\title{
COVID-19 Vaccines: Special Considerations for the Obese Population
}

\author{
Nicholas Kipshidze $^{1}$ (D) $\cdot$ Nodar Kipshidze $^{2} \cdot$ Martin Fried $^{3}$
}

Received: 12 March 2021 / Revised: 27 March 2021 / Accepted: 29 March 2021 / Published online: 8 April 2021

(C) The Author(s), under exclusive licence to Springer Science+Business Media, LLC, part of Springer Nature 2021

It has been demonstrated that obese individuals are at a greater risk for complications following SARS-CoV-2 infection [1]. Beyond COVID-19, obesity has been associated with severe complications from a variety of viral infections. Studies on both seasonal and highly pathogenic influenza show a relationship between complications (i.e., hospitalizations and ICU stays) and increased BMI $[2,3]$. Numerous studies have demonstrated that obesity elicits a state of low-grade chronic inflammation, in part due to the circulation of mononuclear cells including leukocytes and lymphocytes $[4,5]$. Many of the co-morbidities associated with obesity also have shown to alter the immune system, including types 1 and 2 diabetes. Yang and colleagues found that obesity may accelerate thymic involution and a depletion in T-cell repertoire diversity [6]. These effects are typically seen in elderly populations, leading to an increased risk in infection and weakened vaccine efficacy. High fat deposits decrease the level of adiponectin while increasing leptin levels. Adiponectin is an adipocyte hormone, which has shown to decrease macrophage activation and pro-inflammatory cytokine generation, including TNF, IL-6, and $\mathrm{NF}_{\mathrm{k}} \mathrm{B}$ [7]. In contrast, leptin has shown to promote inflammatory reactions in the body as well as its direct action as a pro-inflammatory cytokine [8].

The effect of obesity on vaccine efficacy in humans is not well-studied. Weber and colleagues previously demonstrated the impaired immunogenicity of a now discontinued hepatitis B vaccine in obese patients $[9,10]$. Sheridan and colleagues examined the effects of influenza vaccination in normal, overweight, and obese individuals. While BMI was correlated with a higher initial increase in IgG antibodies post-immunization, at 12 months post-vaccination, higher BMI was associated with a greater decline in antibody titers [11]. Another study

Nicholas Kipshidze

nicholas02@msn.com

NY Cardiovascular Research, New York, NY 10019, USA

2 NYU Langone Health, New York, NY, USA

3 OB Clinic, Prague, Czech Republic demonstrated that BMI was independently associated with inadequate antibody titers 2 years post-rabies vaccination [12].

Current COVID-19 vaccine trials have shown no difference in the vaccine efficacy between normal and obese BMI groups. A pre-print study, however, has demonstrated an association between BMI classes and antibody titers. A stronger humoral response was observed in the groups with underweight and normal-weight vs groups with pre-obesity and obesity participants $(p<0.0001)$. The obese group also demonstrated the widest range in titer levels after the second dose [13]. Therefore, more studies are needed to better understand the long-term efficacy of COVID-19 immunization on obese populations.

Given the negative effects of obesity on the immune system and possible implications in uptake of vaccines, effective and minimally invasive weight loss therapies are needed. Despite the demonstrated efficacy of surgical approaches for weight loss, bariatric surgery could be considered a protective factor with respect to the onset of severe respiratory disease resulting from infection with SARS-CoV-2. The risks of intraprocedural and post-operative complications of bariatric surgery however warrant non-surgical or minimally invasive approaches.

In the midst of the COVID-19 pandemic, more aggressive facilitation of diet, exercise, and behavioral, pharmacologic, non-surgical, and surgical bariatric interventions may be warranted worldwide to [1] reduce complications in obese people exposed to SARS-CoV-2 virus and [2] to maintain efficacy of different COVID-19 vaccines.

Indeed previous studies show a positive association between regular exercise before receiving a flu vaccine and response to the vaccine, producing up to four times as more antibodies after being exposed to a virus or bacteria.

Current advances in pharmaceutical therapies (i.e., semaglutide, tirzepatide, liraglutidue, lorcaeserin, orilistate, and others) could be utilized [14]. Despite the promising response rates of many pharmaceutical therapies, the various side effects as well as a variety of contraindications must also 


\section{COVID-19 Vaccines and Obesity}

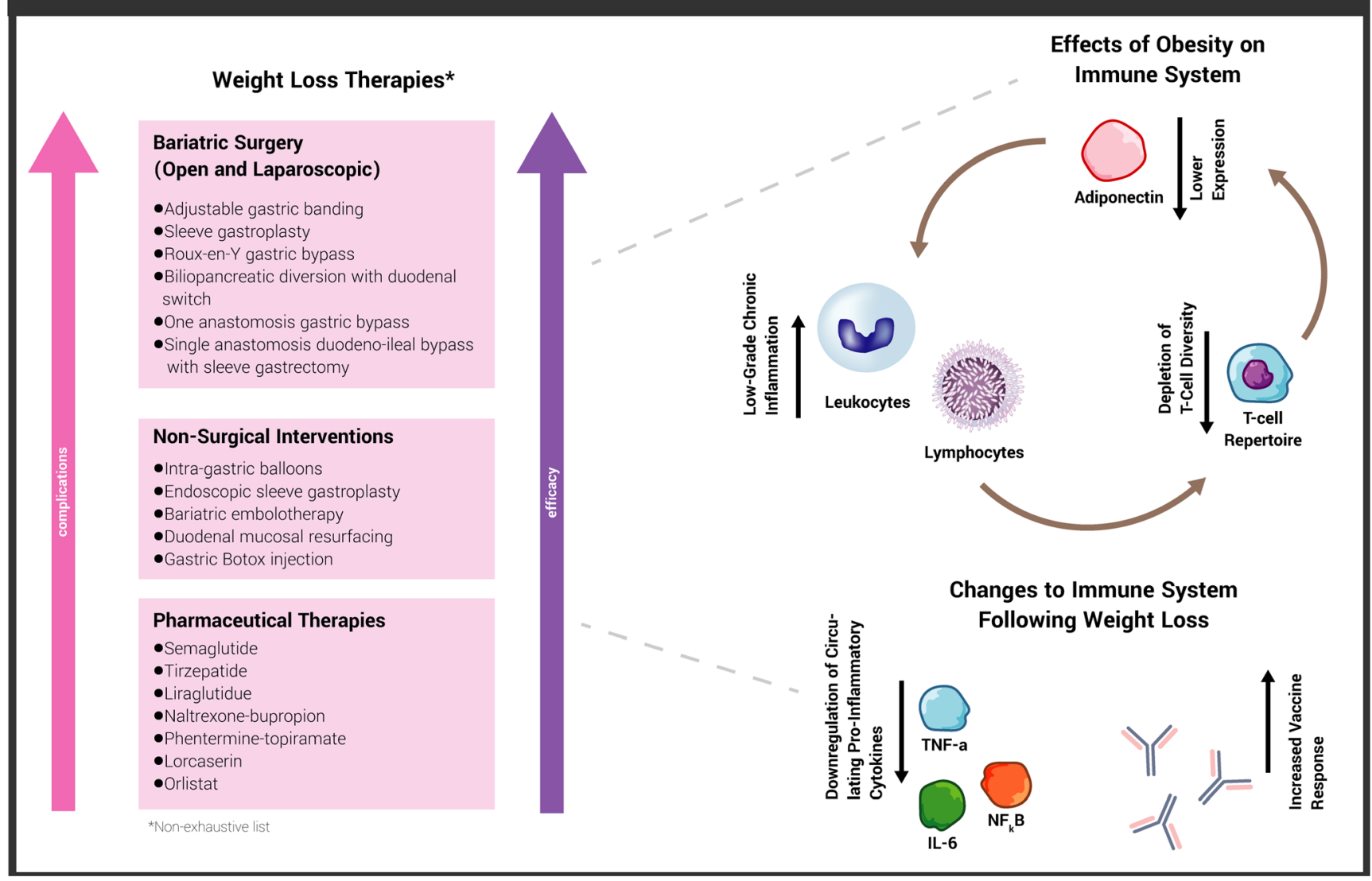

Fig. 1 Approved and investigational weight loss therapies along the risk/benefit spectrum and the effects of obesity and weight loss on the immune system

be considered. The most common events include raised heart rates, renal complications, liver damage, pancreatitis, and gallbladder disease. Additionally, the effects of pharmaceutical therapies on the immune system have not yet been studied.

Finally, non-surgical interventions (intra-gastric balloons, endoscopic sleeve gastroplasty, bariatric embolotherapy, duodenal mucosal resurfacing, gastric botox injection, etc.) can be performed as outpatient procedures, are less risky compared to bariatric surgery, will not compete with hospital beds in overcrowded hospitals, and are powerful tools to achieve rapid and clinically meaningful weight loss. Moreover, it was recently demonstrated that bariatric embolotherapy positively affects the metabolic milieu $[15,16]$. The utilization of these therapies could offer effective (even if limited for a few years) weight loss with a possibly positive impact on lowering the prevalence of serious COVID-19 and enhancing immunogenicity of SARS-CoV-2 vaccines in otherwise compromised obese patients.

Nonetheless, vaccination efforts should continue, and the current vaccine regimen may suffice for obese populations. Ultimately, larger, well-designed studies are needed to better understand the weight-loss effects on both the innate and the adaptive immune system. Additionally, these studies should understand the weight loss thresholds that need to be achieved to initiate positive changes to the immune system.

\section{Declarations}

Conflict of Interest Relationships with industry: NiK is a Founder of Endobar Solutions LLC, and holds stock. NoK is a consultant to Endobar Solutions LLC. MF received research grants from Endobar Solutions LLC.

\section{References}

1. Ng WH, Tipih T, Makoah NA, et al. Comorbidities in SARS-CoV2 patients: a systematic review and meta-analysis. mBio. 2021;12(1)

2. Jain S, Chaves SS. Obesity and influenza. Clin Infect Dis. 2011;53(5):422-4.

3. Kwong JC, Campitelli MA, Rosella LC. Obesity and respiratory hospitalizations during influenza seasons in Ontario, Canada: a cohort study. Clin Infect Dis. 2011;53(5):413-21.

4. Milner JJ, Beck MA. The impact of obesity on the immune response to infection. Proc Nutr Soc. 2012;71(2):298-306. 
5. Nieman DC, Henson DA, Nehlsen-Cannarella SL, et al. Influence of obesity on immune function. J Am Diet Assoc. 1999;99(3):294-9.

6. Yang H, Youm YH, Vandanmagsar B, et al. Obesity accelerates thymic aging. Blood. 2009;114(18):3803-12.

7. Kloting N, Bluher M. Adipocyte dysfunction, inflammation and metabolic syndrome. Rev Endocr Metab Disord. 2014;15(4):27787.

8. Lago F, Dieguez C, Gomez-Reino J, et al. Adipokines as emerging mediators of immune response and inflammation. Nat Clin Pract Rheumatol. 2007;3(12):716-24.

9. Weber DJ, Rutala WA, Samsa GP, et al. Impaired immunogenicity of hepatitis B vaccine in obese persons. N Engl J Med. 1986;314(21):1393.

10. Weber DJ, Rutala WA, Samsa GP, et al. Obesity as a predictor of poor antibody response to hepatitis B plasma vaccine. JAMA. 1985;254(22):3187-9.

11. Sheridan PA, Paich HA, Handy J, et al. Obesity is associated with impaired immune response to influenza vaccination in humans. Int J Obes (Lond). 2012;36(8):1072-7.
12. Banga N, Guss P, Banga A, et al. Incidence and variables associated with inadequate antibody titers after pre-exposure rabies vaccination among veterinary medical students. Vaccine. 2014;32(8):979 83.

13. Pellini R, Venuti A, Pimpinelli F, et al. Obesity may hamper SARSCoV-2 vaccine immunogenisity. medRxiv. 2021.

14. Wadden TA, Bailey TS, Billings LK, et al. Effect of subcutaneous semaglutide vs placebo as an adjunct to intensive behavioral therapy on body weight in adults with overweight or obesity: the STEP 3 randomized clinical trial. JAMA. 2021;

15. Fried M, Kipshidze N, Sramkrova P, et al. Metabolic outcomes of percutaneous transcathether bariatric embolotherapy: insights from an RCT. Obes Surg. 2021.

16. Reddy VY, Neuzil P, Musikantow D, et al. Transcatheter bariatric embolotherapy for weight reduction in obesity. J Am Coll Cardiol. 2020;76(20):2305-17.

Publisher's Note Springer Nature remains neutral with regard to jurisdictional claims in published maps and institutional affiliations. 